\title{
VARIATIONAL PRESSURE IMAGE ASSIMILATION FOR ATMOSPHERIC MOTION ESTIMATION
}

\author{
T. Corpetti ${ }^{1,5}$, P. Héas ${ }^{2}$, E. Mémin ${ }^{1,3}$, N. Papadakis ${ }^{4}$ \\ ${ }^{1}$ IRISA/Universit de Rennes I - Campus de Beaulieu - 35042 Rennes cedex -France, \{name\}@irisa.fr \\ ${ }^{2}$ CEMAGREF - Avenue de Cucillé- 35044 Rennes cedex -France, patrick.heas@cemagref.fr \\ ${ }^{3}$ Fac. de Ing. de la Univ. Buenos-Aires, Av. Paseo Colón 850, C1063ACV Buenos Aires, Argentina \\ ${ }^{4}$ UPF/Barcelona Media, Carrer Ocata 1, 08003 Barcelona - Spain nicolas.papadakis@gmail.com \\ ${ }^{5}$ CNRS / COSTEL UMR 6554, Place du Recteur Henri Le Moal, 35043 Rennes Cedex, France
}

\begin{abstract}
The complexity of dynamical laws governing 3D atmospheric flows associated with incomplete and noisy observations make the recovery of atmospheric dynamics from satellite images sequences very difficult. In this paper, we face the challenging problem of estimating physical sound and time-consistent horizontal motion fields at various atmospheric depths for a whole image sequence. Based on a vertical decomposition of the atmosphere, we propose a dynamically consistent atmospheric motion estimator relying on a multi-layer dynamical model. This estimator is based on a weak constraint variational data assimilation scheme and is applied on noisy and incomplete pressure difference observations derived from satellite images. The dynamical model consists in a simplified vorticity-divergence form of a multi-layer shallow-water model. Average horizontal motion fields are estimated for each layer. The performance of the proposed technique is assessed on real world meteorological satellite image sequences.
\end{abstract}

Index Terms - Data assimilation, pressure images, motion estimation, optical-flow, simplified shallow-water modeling, optimal control theory,

\section{INTRODUCTION}

Geophysical motion characterization and image sequence analysis are crucial issues for numerous scientific domains involved in the study of climate change, weather forecasting and climate prediction or biosphere analysis.

In the context of image-based geophysical motion analysis, standard techniques from computer vision, originally designed for bi-dimensional quasi-rigid motions with stable salient features, appear to be not well adapted [1]. The design of techniques dedicated to fluid flows has been a step forward, towards the constitution of reliable methods to extract characteristic features of flows [2], [3], [4]. However, for geophysical applications, existing fluid-dedicated methods are all limited to frame-to-frame estimation and do not rely on physical conservation laws.
Geophysical flows are quite well described by appropriate physical models. As a consequence, the inclusion of physical evolution laws should constitute a very powerful mean for the motion analysis of satellite image data, in comparison to standard variational or statistical generic image based motion estimation techniques.

Variational data assimilation [5], [6], derived from optimal control theory [7], offers a global optimal formulation allowing combining physical models and different kinds of observations. Since its introduction, the variational assimilation technique commonly known as 4D-Var has been widely used for several atmospheric applications [8], [9]. Most of these methods directly assimilate image data. They do not depend on pseudo-measurements obtained by external techniques but rather propose to include directly a differential observation operator borrowed to image features estimation techniques.

In the same way than [10], the technique proposed in this paper exploits such framework to estimate atmospheric motion directly from satellite image sequences.

\section{DATA ASSIMILATION}

In this section we present the variational data assimilation used for an imperfect dynamical model. We refer the reader to [5], [7], [6] for complete methodological aspects of data assimilation and applications concerning geophysical flows.

The problem consists in recovering, from an initial condition, a system's state $X$ partially observed and driven with an approximately known dynamics. This formalizes as finding $X(\mathbf{s}, t)$, for any location $\mathbf{s}$ at time $t \in\left[t_{0}, t_{f}\right]$, that satisfies the system:

$$
\begin{array}{r}
\frac{\partial X}{\partial t}(\mathbf{s}, t)+\mathbb{M}(X(\mathbf{s}, t))=\nu_{m}(\mathbf{s}) \\
X\left(\mathbf{s}, t_{0}\right)=X_{0}(\mathbf{s})+\nu_{n}(\mathbf{s}) \\
\mathcal{Y}(\mathbf{s}, t)=\mathbb{H}(X(\mathbf{s}, t))+\nu_{o}(\mathbf{s}, t),
\end{array}
$$

where $\mathbb{M}$ is the non-linear operator relative to the dynamics, $X_{0}$ is the initial vector at time $t_{0}$ and $\left(\nu_{n}, \nu_{m}\right)$ are (unknown) additive control variables relative to noise on the dynamics and the initial condition respectively. 
Besides, noisy measurements $\mathcal{Y}$ of the unknown state are available through the non-linear operator $\mathbb{H}$ up to $\nu_{o}$. To estimate the system's state, a common methodology consists in defining a cost-function $\mathcal{J}=$ $\frac{1}{2}\left(\int_{t_{0}}^{t_{f}}\left\|\mathcal{Y}-\mathbb{H}\left(X\left(\nu_{m}, \nu_{n}\right)\right)\right\|_{R^{-1}}^{2} d t+\left\|X\left(\mathbf{s}, t_{0}\right)-X_{0}(\mathbf{s})\right\|_{B^{-1}}^{2}\right.$ $\left.+\int_{t_{0}}^{t_{f}}\left\|\frac{\partial X}{\partial t}(\mathbf{s}, t)+\mathbb{M}(X(\mathbf{s}, t))\right\|_{Q^{-1}}^{2} d t\right)$ to minimize. We have introduced the information matrices $R, B, Q$ relative to the covariance of the errors $\left(\nu_{m}, \nu_{n}, \nu_{o}\right)$. The Mahalanobis distance used reads $\|X\|_{A^{-1}}=X^{T} A^{-1} X$. It can be shown that canceling the gradient $\delta \mathcal{J}_{X}(\theta)$ leads to the following incremental algorithm [11]:

1) Starting from $\tilde{X}\left(\mathbf{s}, t_{0}\right)=X_{0}(\mathbf{s})$, perform a forward integration: $\frac{\partial \tilde{X}}{\partial t}+\mathbb{M}(\tilde{X})=0$

2) $\tilde{X}(\mathbf{s}, t)$ being available, compute the adjoint variables $\lambda(\mathbf{s}, t)$ with the backward equation:

$$
\begin{aligned}
& \boldsymbol{\lambda}\left(t_{f}\right)=0 ; \\
& -\frac{\partial \boldsymbol{\lambda}}{\partial t}(t)+\left(\partial_{X} \mathbb{M}\right)^{*} \boldsymbol{\lambda}(t)=\left(\partial_{X} \mathbb{H}\right)^{*} R^{-1}(\mathcal{Y}-\mathbb{H}(\tilde{X}))(t)
\end{aligned}
$$

3) Update the initial condition : $d X\left(t_{0}\right)=B \boldsymbol{\lambda}\left(t_{0}\right)$;

4) $\lambda$ being available, compute the state space $d X(t)$ from $d X\left(t_{0}\right)$ with the forward integration

$$
\frac{\partial d X}{\partial t}(t)+\left(\frac{\partial \mathbb{M}}{\partial \tilde{X}}\right) d X(t)=Q \boldsymbol{\lambda}(t)
$$

5) Update : $\tilde{X}=\tilde{X}+d X$

6) Loop to step (ii) until convergence

We have here introduced the adjoints variables $\lambda$, the tangent linear operators $\partial \mathbb{M} / \partial \tilde{X}$ and $\partial \mathbb{H} / \partial \tilde{X}$ and their adjoints $\left(\partial_{X} \mathbb{M}\right)^{*}$ and $\left(\partial_{X} \mathbb{H}\right)^{*}$. Intuitively, the adjoints variables $\lambda$ contain information about the discrepancy between the observations and the dynamical model. They are computed from a current solution $\tilde{X}$ with the backward integration (4) that implicates both the observations and the dynamical operators. This deviation indicator between observations and model is then used to refine the initial condition (step 3) and to recover the system state through an imperfect dynamical model where errors are $Q \boldsymbol{\lambda}$ (step 4). If the dynamics is supposed to be perfect, the associated error covariance $Q$ is null and the algorithm only refines the initial condition. However from an image analysis point of view, a perfect modeling appears to be limited since the different models on which we can rely are usually inaccurate due, for instance, to 3D-2D projections, varying lighting conditions, completely unknown boundary conditions at the image boarders, etc.

\section{DATA ASSIMILATION FOR MULTI-LAYER SHALLOW-WATER MODEL}

In this section, the variational assimilation framework is used to estimate atmospheric motion fields from sparse pressure difference images.

Input data: we deal with top of cloud pressure images of kilometer order that are routinely provided by the EUMETSAT consortium. From a set of Meteosat Second
Generation (MSG) channels, sparse top of cloud pressure images related to the different layers of the atmosphere are derived from a radiative transfer model using ancillary data, namely temperature and humidity profiles obtained by analyzes on short term forecasts (see [12], [13] for details). We denote by $C^{k}$ the class corresponding to the $k$-th layer. Some image are shown in figure 1 for 2 levels of the atmosphere.

Dynamics As satellite images supply only sparse data of partially visible 3D cloud layers for which only inaccurate pressure vertical coordinates can be indirectly inferred, we focus in this work on the simpler task of estimating integrated horizontal wind fields over successive atmospheric layers. The layering of atmospheric flow in the troposphere is valid in the limit of horizontal scales much greater than the vertical scale height. Thus for layers of a thickness on the order of scale of $1 \mathrm{~km}$, this hypothesis is roughly valid for horizontal scales greater or equal to $100 \mathrm{~km}$. It is thus impossible to truly characterize a layered atmosphere with a local analysis performed in the vicinity of a pixel characterizing a kilometer order scale. We then filter the Hydrostatic Primitive Equations (HPE) with a Gaussian kernel function $K_{\delta_{x}}$, neglect their vertical derivatives and consider that filtered horizontal winds $\tilde{\mathbf{v}}^{k}$, which have been vertically averaged, are equal to filtered horizontal winds on layer upper surfaces $\mathbf{s}^{k+1}$. Starting from the HPE under these assumptions yields to (see [14] for details):

$\tilde{\mathbf{v}}_{t}^{k}+\nabla\left(\tilde{\mathbf{v}}^{k}\right) \tilde{\mathbf{v}}^{k}-\rho_{0}^{-1} \nabla \tilde{p}\left(s^{k+1}\right)+\left[\begin{array}{cc}0 & -1 \\ 1 & 0\end{array}\right] f^{\phi} \tilde{\mathbf{v}}^{k}=\nu_{\mathcal{T}} \boldsymbol{\Delta}\left(\tilde{\mathbf{v}}^{\mathbf{k}}\right)$,

where $\tilde{\mathbf{v}}^{\mathbf{k}}=\left(u^{k}, v^{k}\right)^{T}$ denotes the horizontal velocity field for the layer $k, \rho_{0}$ the density, $\tilde{p}$ the pressure, $f^{\phi}$ the coriolis force, $\nu_{\mathcal{T}}$ a diffusion coefficient and $\nabla\left(\tilde{\mathbf{v}}^{\mathbf{k}}\right)=$ $\left(\boldsymbol{\nabla} \tilde{u}^{k}, \boldsymbol{\nabla} \tilde{v}^{k}\right)^{\top}$ and $\boldsymbol{\Delta}\left(\tilde{\mathbf{v}}^{\mathbf{k}}\right)=\left(\Delta \tilde{u}^{k}, \Delta \tilde{v}^{k}\right)^{\top}$. Let us denote the vorticity by $\tilde{\zeta}^{k}=\operatorname{curl}\left(\tilde{\mathbf{v}}^{k}\right)$ and the divergence by $\tilde{D}^{k}=\operatorname{div}\left(\tilde{\mathbf{v}}^{k}\right)$. The previous system may be expressed in its vorticity-divergence form:

$$
\left\{\begin{array}{l}
\tilde{\zeta}_{t}^{k}+\tilde{\mathbf{v}}^{k} \cdot \nabla \tilde{\zeta}^{k}+\left(\tilde{\zeta}^{k}+f^{\phi}\right) \tilde{D}^{k}=\nu_{\mathcal{T}} \Delta\left(\tilde{\zeta}^{k}\right), \\
\tilde{D}_{t}^{k}+\tilde{\mathbf{v}}^{k} \cdot \nabla \tilde{D}^{k}+\left(\tilde{D}^{k}\right)^{2}-2|J|-\rho_{0}^{-1} \Delta \tilde{p}\left(s^{k+1}\right)+f^{\phi} \tilde{\zeta}^{k}= \\
\nu_{\mathcal{T}} \Delta\left(\tilde{D}^{k}\right)
\end{array}\right.
$$

where $|J|$ is the determinant of the Jacobian matrix of variables $\left(\tilde{u}^{k}, \tilde{v}^{k}\right)$. For vorticity based large eddy simulation formulations, we may rely on enstrophy-based sub-grid models [15]. This sub-grid dissipation model is based on Taylor's vorticity transfer and dissipation by small scales theory ([16]) and reads: $\nu_{\mathcal{T}}=\left(C \delta_{x}\right)^{2}\left|\tilde{\zeta}^{k}\right|$. In the momentum conservation formulations of equation (6) and equation (7), dynamical models predict the evolution of velocity components $\left(\tilde{u}^{k}, \tilde{v}^{k}\right)$ and of divergence and vorticity $\left(\tilde{\zeta}^{k}, \tilde{D}^{k}\right)$. In both models, one of the major difficulties is induced by the dependence to the pressure variable $\tilde{p}\left(s^{k+1}\right)$ which is an unknown variable of the $k$-th layer state. Thus, we search instead to derive a dynamical model which is independent of the pressure unknown. Opposite to the classical formulation, the vorticity-divergence equations have the advantage of providing such a model for the vorticity evolution. As regards the divergence, since at large scales, it can be considered 
weak almost everywhere, we will rely on an approximate evolution law. We assume here that the divergence is advected by the flow and a noise variable that encodes the uncertainty on the model. More precisely we will assume that the divergence map is a function of a stochastic process representing a particle position and is driven by the following stochastic differential equation:

$$
d \mathbf{x}(t)=\tilde{\mathbf{v}}^{k}(\mathbf{x}(t)) d t+\sqrt{2 \nu_{T}} d B(t) .
$$

This equation states that the particle position is known only up to an uncertainty that grows linearly with time. Here $B_{t}$ denotes a standard Brownian motion of $\mathbb{R}^{2}$. The process $\mathbf{x}_{t}$ starts at point, $\boldsymbol{x}_{o}$. After some manipulations detailed in [14] (through the Ito formula, the Kolmogorov's forward equation and assuming that the divergence of the flow is given by its expectation), the simplified filtered vorticity-divergence model for the layer $k$ can be defined:

$$
\left\{\begin{array}{l}
\tilde{\zeta}_{t}^{k}+\tilde{\mathbf{v}}^{k} \cdot \nabla \tilde{\zeta}^{k}+\left(\tilde{\zeta}^{k}+f^{\phi}\right) \tilde{D}^{k}=\nu_{\mathcal{T}} \Delta \tilde{\zeta}^{k} \\
\tilde{D}_{t}^{k}+\tilde{\mathbf{v}}^{k} \cdot \nabla \tilde{D}^{k}+\left(\tilde{D}^{k}\right)^{2}=\nu_{\mathcal{T}} \Delta \tilde{D}^{k}
\end{array}\right.
$$

In this model we assume that the divergence of the flow is weak and is similar to the divergence expectation. The divergence equation does not describe anymore the evolution of the flow divergence but the evolution of its expectation. The expectation of the divergence value is advected by the flow and dissipates due to a subgrid isotropic uncertainty. This hypothesis is quite natural in large scales modeling. The model in (9) constitutes our dynamical model for the assimilation process.

State variables We choose to represent the system state $X$ (i.e. the velocity field) through the curl and divergence components: $X=\left[\tilde{\zeta}^{k}, \tilde{D}^{k}\right]^{T}$ as their evolution can be described by equation (9) and since they completely determine the underlying 2D velocity up to a harmonic transportation component. As shown in [14], the field can be represented by its div-curl components as $\tilde{\mathbf{v}}^{k}=\nabla^{\perp}\left(G * \tilde{\zeta}^{k}\right)+\nabla\left(G * \tilde{D}^{k}\right)=$ $\underbrace{\left[\nabla^{\perp} G *, \nabla G *\right]}_{\mathbb{H}_{G}} \underbrace{\left[\begin{array}{c}\tilde{\zeta}^{k} \\ \tilde{D}^{k}\end{array}\right]}_{X}$, where $G$ is the 2D Green kernel associated to the Laplacian operator and where $\mathbb{H}_{G}$ can efficiently be computed in the Fourier domain.

Image observation operator In order to define an observation operator that links the unknown motion field $\tilde{\mathbf{v}}^{k}$ to images of pressure differences $h_{o b s}^{k}(\mathbf{s}, t)$, we use the mass conservation law of equation:

$$
\frac{\partial h_{o b s}^{k}(\mathbf{s}, t)}{\partial t}+\nabla h_{o b s}^{k}(\mathbf{s}, t) \cdot \tilde{\mathbf{v}}^{k}(\mathbf{s}, t)+h_{o b s}^{k}(\mathbf{s}, t) \operatorname{div} \tilde{\mathbf{v}}^{k}(\mathbf{s}, t) \approx 0,
$$

However, this formulation cannot be used alone to estimate $\tilde{\mathbf{v}}^{k}$ as it provides only one equation for two unknowns at each spatio-temporal location $(\mathbf{s}, t)$, with therefore a onedimensional family of solutions in general. To remove such ambiguities, a common approach consists in assuming a spatial coherence of wind field estimates in a given neighborhood (similar to the well-known approach of [17] used in computer vision). In the present case, this assumption is coherent since unknowns are velocity vectors $\tilde{\mathbf{v}}^{k}(\mathbf{s}, t)$ that are spatially filtered within a neighborhood of size $\delta_{x}$. The measured motion field should thus obey to the constraint:

$$
K_{\delta_{x}} *\left(\frac{\partial h_{o b s}^{k}}{\partial t}+\nabla h_{o b s}^{k} \cdot \tilde{\mathbf{v}}+h_{o b s}^{k} \operatorname{div} \tilde{\mathbf{v}}^{k}\right) \approx 0,
$$

where $K_{\delta_{x}}$ is the Gaussian kernel of standard deviation $\delta_{x}$. Recalling that $\tilde{\mathbf{v}}^{k}=\mathbb{H}_{G} X$ (with $X=\left[\tilde{\zeta}^{k}, \tilde{D}^{k}\right]^{T}$ ), one can easily define our observation system $\mathcal{Y}=\mathbb{H}(X)$ with $\mathcal{Y}=$ $K_{\delta_{x}} * \frac{\partial h_{o b s}^{k}(\mathbf{s}, t)}{\partial t}$ and $\mathbb{H}(X)=-\left(K_{\delta_{x}} * \nabla h_{o b s}^{k}\right)^{T} \mathbb{H}_{G}(X)-$ $\left(K_{\delta_{x}} * h_{o b s}^{k}\right) \nabla^{T} \mathbb{H}_{G}(X)$.

\section{EXPERIMENTS}

We present a qualitative evaluation of our method on MSG image sequences acquired on north of Atlantic Ocean during part of one day (5-June-2004) from $13 \mathrm{~h} 30$ to $15 \mathrm{~h} 45$ UTC at a rate of an image every 15 minutes. This benchmark data is composed of 10 frames sequences of top of cloud pressure and cloud-classification images. The image spatial resolution is $3 \times 3 \mathrm{~km}^{2}$ at the center of the whole Earth image disk. The cloud-classifications were used to segment images into $K=3$ broad layers, at low, intermediate and high altitude. In figure 1 we present a sample of the motion fields estimated for two different layers together with the original observations. The motion fields estimated for the different layers are consistent with a visual inspection of the sequence. In particular, several motion differences between layers are very relevant. For instance, near the bottom left corner of the images, the lower layer possesses a southward motion while the intermediate layer moves northward. A complete evaluation can be founded in [14].

\section{CONCLUSION}

In this paper, we have proposed an assimilation approach enabling, for the first time, a dynamically consistent estimation of a sequence of dense and layered atmospheric wind fields from an entire satellite image sequences. The motion estimator is applied to sparse pressure difference images corresponding to a stack of layers in a stratified atmosphere. A method was proposed to derive such images from top of cloud pressure images and classification, which are routinely supplied by the EUMETSAT consortium. The dynamical constraint of our system suggested relying on a simplification of the vorticity divergence form of a shallowwater dynamics for which the momentum equations are independent of the layers thickness. The mass conservation law complements this dynamics and provides the basis of an original image-adapted observation operator.

\section{REFERENCES}

[1] B. Horn and B. Schunck, "Determining optical flow," Artificial Intelligence, vol. 17, pp. 185-203, 1981.

[2] T. Corpetti, D. Heitz, G. Arroyo, E. Mémin, and A. Santa-Cruz, "Fluid experimental flow estimation based on an optical-flow scheme," Experiments in fluids, vol. 40, pp. 80-97, 2006.

[3] A. Cuzol and E. Mémin, "A stochastic filter technique for fluid flows velocity fields tracking," IEEE Trans. Pattern Anal. Machine Intell., 2008, accept for publication.

[4] J. Yuan, C. Schnoerr, and E. Mémin, "Discrete orthogonal decomposition and variational fluid flow estimation," J. of Math. Imaging and Vision, vol. 28, no. 1, pp. 67-80, 2007. 

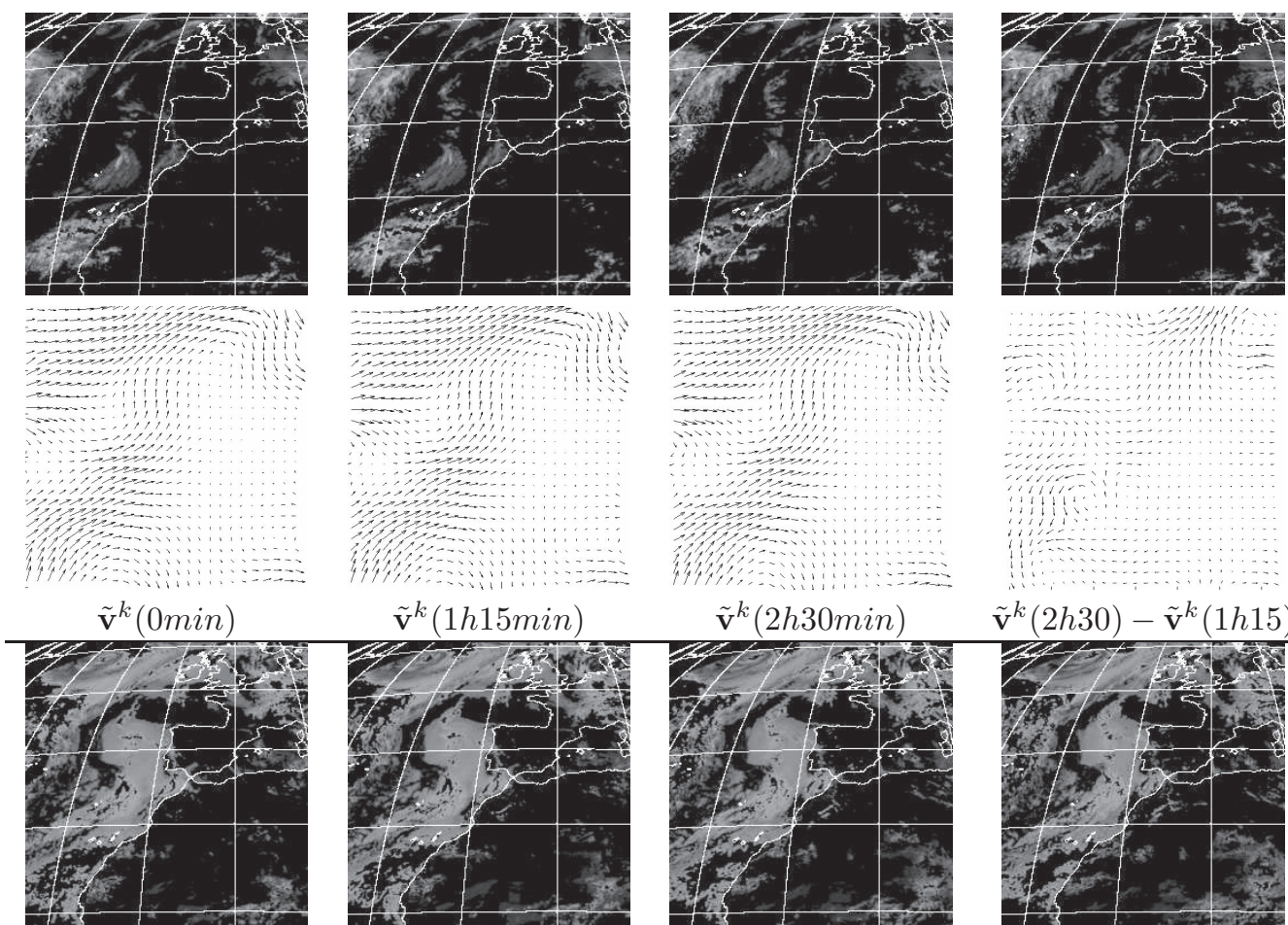

$\tilde{\mathbf{v}}^{k}(2 h 30)-\tilde{\mathbf{v}}^{k}(1 h 15)$
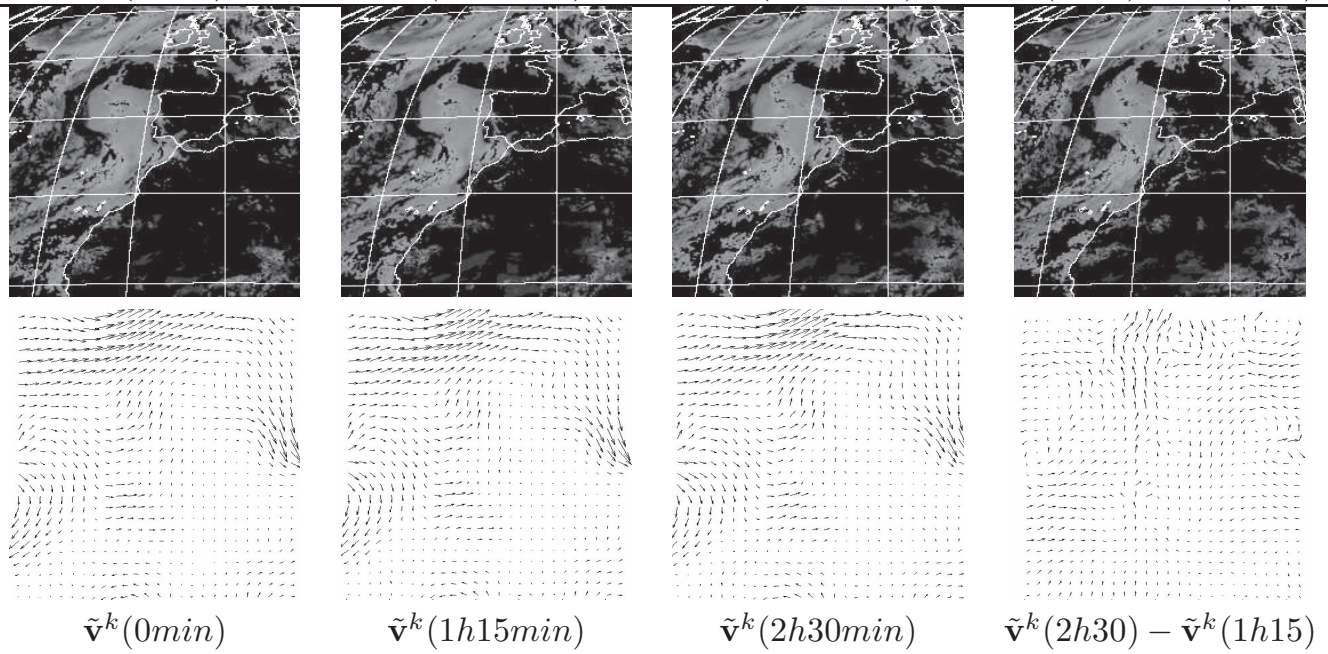

Fig. 1. Horizontal wind fields estimated by assimilation with an imperfect modeling for the intermediate layer (top part) and the lower layer (bottom part). For each layer, the first line represents 4 images of the sequences. The three first motion fields of the second line corresponds to estimation at $t=0 \mathrm{~min}, t=1 \mathrm{~h} 15 \mathrm{~min}$ and $t=2 \mathrm{~h} 30 \mathrm{~min}$ respectively. The last motion field is the difference $\tilde{\mathbf{v}}^{k}(2 h 30)-\tilde{\mathbf{v}}^{k}(1 h 15)$ and illustrates the temporal changes.

[5] F.-X. Le Dimet and O. Talagrand, "Variational algorithms for analysis and assimilation of meteorological observations: theoretical aspects," Tellus, pp. 97-110, 1986.

[6] O. Talagrand and P. Courtier, "Variational assimilation of meteorological observations with the adjoint vorticity equation. I: Theory," $J$. of Roy. Meteo. soc., vol. 113, pp. 1311-1328, 1987.

[7] J.L. Lions, Optimal control of systems governed by PDEs, SpringerVerlag, 1971.

[8] A.F. Bennet, Inverse Methods in Physical Oceanography, Cambridge University Press, 1992.

[9] O. Talagrand, "Assimilation of observations, an introduction," $J$. Meteor. Soc. Jap., vol. 75, pp. 191-209, 1997.

[10] N. Papadakis and E. Mémin, "A variational technique for time consistent tracking of curves and motion," Journal of Mathematical Imaging and Vision, vol. 31, no. 1, pp. 81-103, May 2008.

[11] A.F. Bennett and M.A. Thorburn, "The generalized inverse of a nonlinear quasigeotrophic ocean circulation model.," J. Phys. Ocean.,, vol. 22, pp. 213-230, 1992.

[12] P. Héas, E. Mémin, N. Papadakis, and A. Szantai, "Layered estimation of atmospheric mesoscale dynamics from satellite imagery," IEEE
Trans. Geoscience and Remote Sensing, vol. 45, no. 12, pp. 40874104, 2007.

[13] H.J. Lutz, "Cloud processing for meteosat second generation," Tech Rep., European Organisation for the Exploitation of Meteorological Satellites (EUMETSAT), Available at : http://www.eumetsat.de, 1999.

[14] T. Corpetti, P. Héas, E. Mémin, and N. Papadakis, "Pressure image assimilation for atmospheric motion estimation," Research Report 6507, INRIA, 042008.

[15] N. N. Mansour, J. H. Ferziger, and W. C. Reynolds, "Large-eddy simulation of a turbulent mixing layer," Tech. Rep., Report TF11, Thermosciences Div., Dept. of Mech. Eng., Standford University, 1978.

[16] GI. Taylor, "The transport of vorticity and heat through fluids in turbulent motion," in Proc London Math Soc. Ser A, 1932, pp. 151421.

[17] B. Lucas and T. Kanade, "An iterative image registration technique with an application to stereovision," in Int. Joint Conf. on Artificial Intel. (IJCAI), 1981, pp. 674-679. 